\title{
EDITORIAL
}

\section{NG2 antigen is expressed in CD34 + HPCs and plasmacytoid dendritic cell precursors: is NG2 expression in leukemia dependent on the target cell where leukemogenesis is triggered?}

Leukemia (2008) 22, 1475-1478; doi:10.1038/leu.2008.134

The neuron-glial antigen 2 (NG2) molecule and its human homolog was first reported on oligodendrocyte progenitor cells. ${ }^{1}$ NG2 is recognized by the 7.1 monoclonal antibody. ${ }^{2}$ The physiological role of this molecule remains to be elucidated. ${ }^{3}$ The expression pattern of NG2 in leukemia is controversial. NG2 expression was initially claimed to be specifically associated with 11q23/mixed-lineage leukemia (MLL) gene rearrangements. ${ }^{4}$ In fact, over the past years, NG2 has been incorporated in diagnostic panels for immunophenotyping of leukemic patients because of its potential predictive value for MLL rearrangements in childhood and adult acute myeloid leukemias. ${ }^{2,4-9}$ However, it has been suggested that 7.1 expression could be specifically associated with only two specific subtypes of leukemia harboring either the translocations $\mathrm{t}(4 ; 11)(\mathrm{q} 21 ; \mathrm{q} 23)$ or $\mathrm{t}(9 ; 11)(\mathrm{p} 13 ; \mathrm{q} 23)$, which encode for the leukemic fusion genes MLL-AF4 and MLL-AF9, respectively, but not to other MLL rearrangements. ${ }^{10}$ Moreover, in the clinic, many leukemic patients harboring MLL rearrangements but lacking NG2 expression are commonly seen (Supplementary Table 1). In turn, we and many others have reported the existence of acute leukemias and plasmacytoid dendritic cell (pDC) leukemias (>50\%) lacking MLL rearrangements but expressing NG2 ${ }^{11}$ (Supplementary Table 1).

Over 80 different partners of the human MLL gene have been identified so far, most being associated with a poor clinical outcome. ${ }^{12-14}$ Clearly, this renders the characterization of MLL fusion alleles and its correlation with NG2 expression a very demanding task. Intriguingly, it has been suggested that the expression of NG2 antigen may be dependent on the particular gene(s) paired to MLL when it is rearranged, explaining, at least in part, why some patients with 11 q23 balanced translocations can be NG2 negative. ${ }^{5,10}$

On the basis of the controversial data about the clinical relevance of NG2 expression and its inclusion in diagnostic immunophenotypic panels as well as the existence of NG2expressing acute leukemias lacking MLL rearrangements, in particular pDC leukemias, ${ }^{11}$ we aimed (i) to gain further insights into the biological association between NG2 expression and MLL rearrangements; (ii) to analyze whether the expression of NG2 may depend on the particular gene(s) paired to MLL when it is rearranged and (iii) to explore the hypothesis that the expression of NG2 in leukemias lacking MLL rearrangements, such as NG2 + pDC-leukemias, may be due to the existence of a minor subset of CD34+ hematopoietic stem/progenitor cells readily coexpressing NG2 where the leukomogenesis process may be initially triggered.

The following cell lines, chosen based on the status of the MLL locus were used in this study: KG1a (stem cell like-AML), REH (TEL-AML1 + pre-B ALL), 293T (kidney embryonic epithelial cell line), MV4;11 (MLL-AF4 + pro-B ALL), RS4;11 (MLL-AF4 + pro-B ALL) and THP-1 (MLL-AF9 + AML). Three out of the six (KG1a, REH and 293T) are cell lines without MLL translocations, whereas the other three cell lines $(\mathrm{MV} 4 ; 11$, RS4;11 and THP-1) harbor balanced MLL translocations. Importantly, AF4 is the partner gene paired to rearranged MLL in both MV4;11 and RS4;11 cell lines, whereas AF9 is paired to MLL in THP-1. The cell lines were maintained in standard culture conditions: RPMI or DMEM with $10 \%$ fetal bovine serum supplemented with nonessential amino acids, $2 \mathrm{mM}$ L-glutamine and antibiotics (all from Gibco, Invitrogen, Grand Island, NY, USA). Genetically stable (manuscript submitted) and fully characterized pluripotent undifferentiated human embryonic stem cells (hESCs) HS181 (kindly provided by Professor O Hovatta, Karolinska Institute) were maintained on mitotically inactivated human embryonic fibroblasts as described previously. ${ }^{15}$ Cord blood samples (CB; $n=5)$ from healthy newborns were obtained from local hospitals with Ethics Board approval. Normal bone marrow $(n=6)$ and granulocyte-colony stimulating factor (G-CSF)-mobilized peripheral blood $(n=1)$ were obtained from healthy volunteers upon informed consent. The mononuclear cells were isolated using Ficoll-Hypaque (GE Healthcare, Bucks, UK), washed with phosphate-buffered saline $+3 \%$ fetal bovine serum and the red blood cells lysed (Red Blood Cell-Lysis Solution, BD Biosciences, Erembodegem, Belgium). Then, the mononuclear cells were stained by direct immunofluorescence (see below) and ready for multicolor high-speed flow cytometry analysis. ${ }^{16}$

The expression of NG2 in CD34 + pDC precursors ${ }^{16}$ was assessed by flow cytometry using the following combination of monoclonal antibody in a six-color staining: CD38-FITC/NG2PE/HLADR-PerCP/CD123-APC/CD34-PECy7/CD45-APC-Cy7. All monoclonal antibody were purchased from Becton Dickinson (San Jose, CA, USA) except the NG2 that is from Immunotech (Marseille, France) and CD123-APC from Miltenyi (Bergisch, Gladbach, Germany). Of note, as many as $2 \times 10^{5}$ CD34 + hematopoietic stem/progenitor cells were gated and analyzed. Plasmacytoid DC precursors were identified as being $\mathrm{CD} 4^{+} / \mathrm{CD} 45^{+} / \mathrm{CD} 38^{+} / \mathrm{CD} 123^{\text {high }} / \mathrm{HLADR}^{+}$, as described recently. ${ }^{16}$ From an ontogeny standpoint, the expression of NG2 was assessed in hESCs (prenatal stage of in utero development) and CB samples (neonatal stage of hematopoietic development), because a high frequency of primary leukemias with rearranged MLL gene occurs during infancy/childhood. ${ }^{17}$ NG2 expression was also analyzed in adult bone marrow samples.

Among the cell lines without MLL rearrangements, the myeloid cell line KG1a lacked NG2 expression, whereas REH was NG2 + (Figure 1a). Interestingly, the nonhematopoietic cell line 293T was also clearly positive for NG2. This data confirm the expression of NG2 in hematopoietic and nonhematopoietic cell lines without MLL rearrangements, further supporting the existence of human leukemias lacking MLL translocations but expressing the NG2 antigen ${ }^{11}$ (Figure 1a and Supplementary Table 1).

Among the MLL translocation-harboring cell lines, THP-1 displayed clear expression of NG2. Intriguingly, both the 

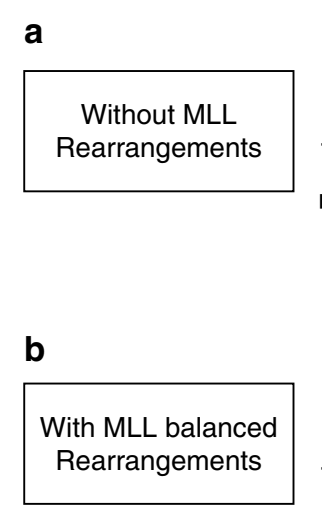
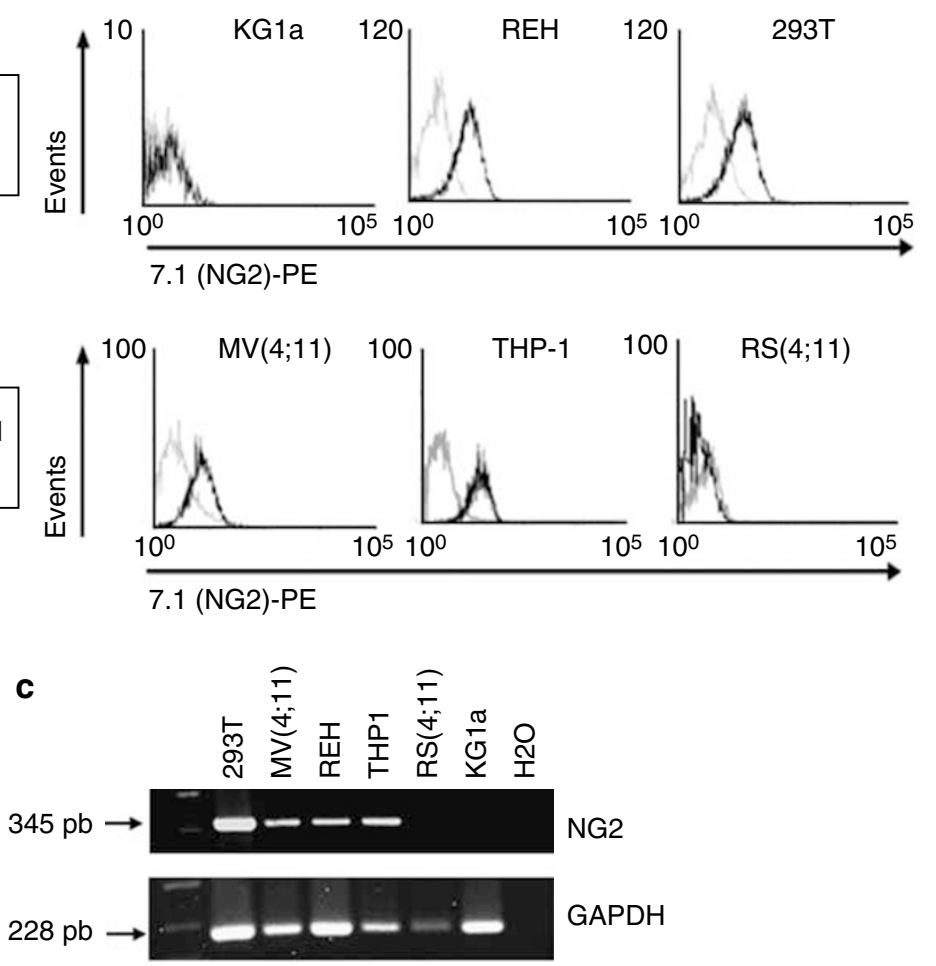

Figure 1 Flow cytometry analysis of NG2 (7.1) antigen in transformed hematopoietic cell lines. (a) Expression of NG2 in transformed cell lines lacking MLL gene rearrangements. (b) Expression of NG2 in transformed cell lines carrying balanced MLL gene rearrangements. Gray lines represent the irrelevant isotype-matched control. (c) reverse transcriptase showing the NG2 mRNA expression in different leukemic cell lines. MLL, mixed-lineage leukemia; NG2, neuron-glial antigen 2.

MV4;11 and RS4;11 cell lines, while sharing MLL gene rearrangements fused to the same partner gene (AF4), displayed a different profile of NG2 expression: MV4;11 cells were NG2positive, whereas RS4;11 were negative (Figure 1b). To add more mechanistic insight, NG2 expression was also analyzed at the RNA level by reverse transcriptase-PCR. As shown in Figure 1c, NG2 mRNA is present in all the NG2 + leukemic cell lines and absent in all the NG2 - leukemic cell lines, indicating a full correlation between NG2 mRNA detection and NG2 transcribed protein.

Our experimental data support the clinical finding of both primary human leukemias with balanced MLL rearrangements coexpressing NG2 and human leukemia cases harboring balanced MLL gene translocations but lacking NG2 expression (Supplementary Table 1). This data not only highlight the lack of association between NG2 antigen and MLL rearrangements but also provide preliminary experimental evidence against the hypothesis that NG2 expression in human leukemia may be dependent on the particular gene(s) paired to MLL when rearranged, as we demonstrate that two pro-B ALL cell lines (MV4;11 and RS4;11) with the MLL gene fused to the same partner, AF4, have a completely different NG2 antigen expression profile (Figure 1).

Several cellular and molecular mechanisms, intrinsic molecular determinants and extrinsic signals may contribute to the controversial correlation between MLL rearrangements and NG2 regulation. The confused association observed in actual leukemic samples makes an explanation based on a potential role for 'in vivo' (bone marrow, $\mathrm{CB}$ and so on) occurring cell-cell interactions unlikely. Similarly, our 'in vitro' data, rule out the possibility that potential hits/mutations secondary to MLL translocations are required for triggering NG2 expression, as we used fully transformed/immortalized cell lines derived from patients with overt disease, therefore carrying a paramount of cooperating mutations and genetic insults. The possibility that NG2 expression could be associated with nonbalanced MLL rearrangements such as deletions or inversions has previously been ruled out. ${ }^{4,5}$ Although unlikely, the possibility that NG2 expression could be linked to MLL internal duplications, which are not prospectively analyzed in human leukemias at diagnosis, should not be excluded.

Here, we hypothesize that NG2 expression may be dependent on the cell of origin where a specific leukemic abnormality initially occurs. For instance, NG2 might only be regulated when the leukemic abnormality arises either in a lineagespecific progenitor (hematopoietic progenitor cell; HPC) or in a more immature, less committed stem cell (hematopoietic stem cell; HSC). When a large number $\left(2 \times 10^{5}\right.$ cells $)$ of CD34+ hematopoietic stem/progenitor cells are gated and analyzed by flow cytometry, coexpression of NG2 is readily observed in a subset of CD34+CD38+ HPCs from CB $(2.1 \pm 2.4 \%$; Figure $2 \mathrm{a})$, bone marrow $(0.83 \pm 2.2 \%)$ and mobilized peripheral blood (1.3\%; Supplementary Figure 1a), suggesting that HPCs rather than HSCs may be the target cell for transformation. To verify that this CD34+CD38+NG2 + cell subset represents truly HPCs, this population was enriched (74\% post-sort purity) by flurescence-activated cell sorting from $\mathrm{CB}$ and the cells plated in methylcellulose assays. Importantly, multilineage (CFU-G, CFU-M; CFU-Mix, BFU-E) hematopoietic colonies were obtained in in vitro colony-forming unit (CFU) assays ${ }^{18}$ (Supplementary Figure 1a), suggesting that HPCs rather than HSCs may be the target cell for transformation. Recent studies from Vormoor laboratory ${ }^{19,20}$ support our data as they found that NG2 is not expressed in MLL-rearranged HSC populations $($ CD34 + CD38-) but was up-regulated in differentiated MLL- 

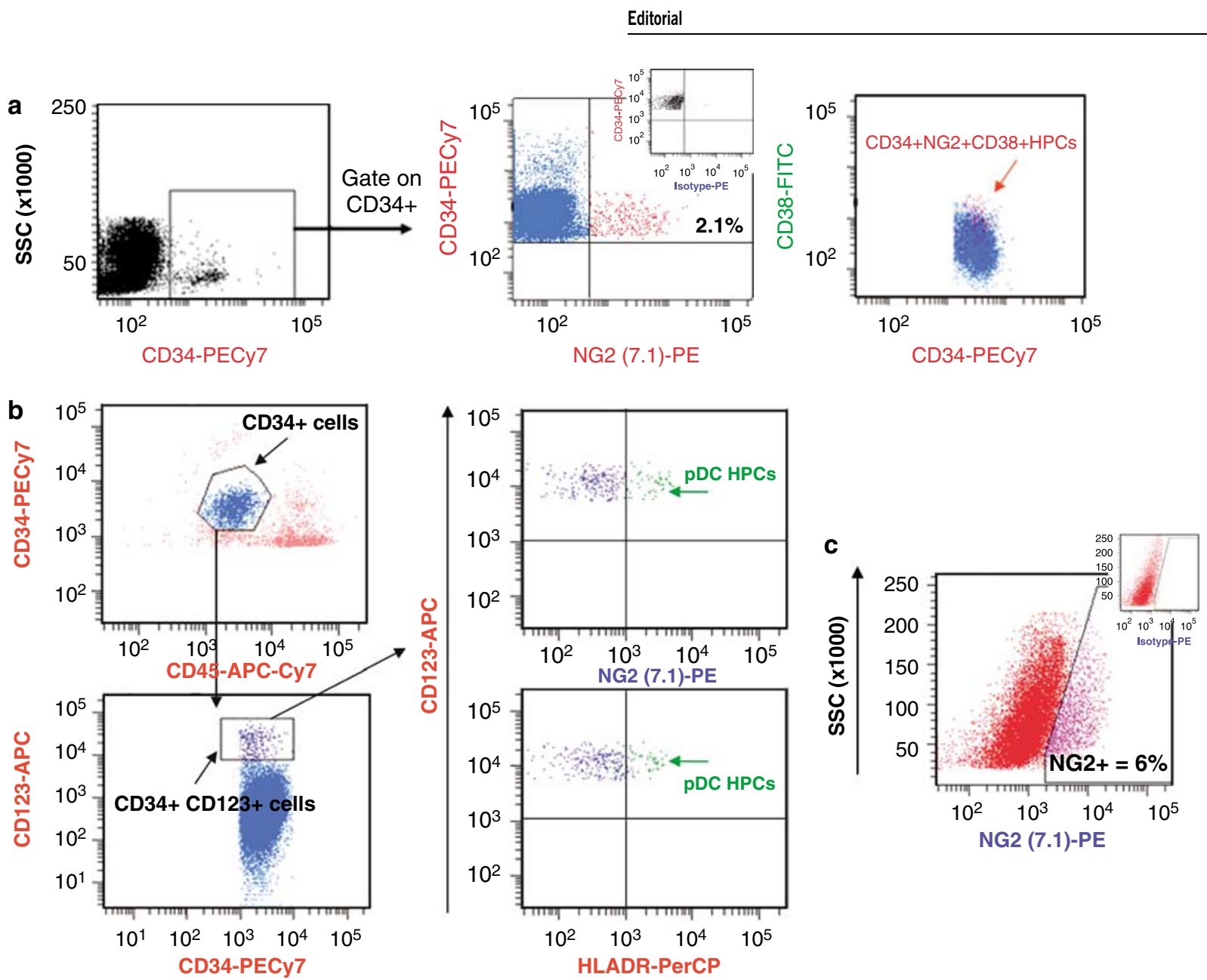

Figure 2 Representative flow cytometry analysis of NG2 (7.1) antigen in normal CD34 + progenitors, CD34 + pDC precursors and hESCs. (a) Expression of NG2 in gated CB-derived CD34+ cells. Analyses of as many as $2 \times 10^{5} \mathrm{CD} 34+$ cells revealed that $2.1 \pm 2.4 \%$ of the CD34 + cells coexpress NG2 + cells. All these CD34 + NG2 + cells are CD38 +, therefore, representing HPCs. An irrelevant isotype-matched antibody was used as a negative control (inset panel). (b) Six-color high-speed flow cytometry analysis showing the expression of NG2 (green dots) in a population of pDC precursors (CD45 + CD34 + CD38 + CD123 + + and HLADR +$){ }^{16}(\mathbf{c})$ Representative NG2 expression in genetically stable hESCs. An irrelevant isotype-matched antibody was used as a negative control (inset panel). CB, cord blood; HPCs, hematopoietic progenitor cells; NG2, neuron-glial antigen 2; pDC, plasmacytoid dendritic cell.

rearranged leukemic blasts $(\mathrm{CD} 19+\mathrm{B}$-lineage blasts and $\mathrm{CD} 33+$ myeloid blasts) in pediatric MLL-rearranged AMLs and MLL-AF4 + ALLs.

The expression of NG2 in $60 \%$ of pDC leukemias lacking MLL rearrangements have recently been reported ${ }^{11}$ (our unpublished observations). We, therefore, next addressed whether pDC $\mathrm{CD} 34+$ precursors $^{16}$ coexpress NG2. Interestingly, we found that $12.2 \pm 13 \%$ of the pDC precursors $\left(\mathrm{CD} 34^{+} / \mathrm{CD} 45^{+} /\right.$ $\mathrm{CD} 8^{+} / \mathrm{CD} 123^{\text {high }} / \mathrm{HLADR}^{+}$) readily coexpress NG2 (Figure 2b). This suggests that, regardless of the status of the MLL locus, the NG2 antigen may be expressed in pDC leukemias, if the leukomogenesis process is initially triggered in a pDC $\mathrm{CD} 34+$ precursor readily expressing NG2, which might act as a leukemic-initiating cell. In fact, based on the existence of $\mathrm{NG} 2+$ cell lines and $\mathrm{NG} 2+$ human primary leukemias ${ }^{11}$ (our unpublished observations) lacking MLL rearrangements our data illustrate that the leukemic abnormality underlying NG2 expression does not necessarily need to be an MLL rearrangement.

Unlike other MLL fusion proteins, MLL-AF4 fusion gene resulting from the $t(4 ; 11)$ is always found in infant pro-B-ALL with a dismal prognosis and arises prenatally. ${ }^{17}$ From an ontogeny standpoint, two possible scenarios have been proposed: (i) MLL-AF4 arises and has its preleukemic impact during early human embryonic hematopoiesis or (ii) MLL-AF4 arises during early hematopoiesis but has its preleukemic impact only in later fetal hematopoiesis. ${ }^{17}$ We analyzed the expression of NG2 in undifferentiated hESCs, a potential prenatal target cell for MLL-AF4 occurrence. Intriguingly, NG2 expression is also readily found in $5-6 \%$ of genetically stable hESCs (Figure $2 d$ ).

Despite NG2 and its human homolog was first reported on oligodendrocyte progenitor cells, the possibility that the cell subset CD34 + NG2 + might represent a potential circulating neural progenitors should be ruled out since, as shown by sixcolor high-speed flow cytometry, the NG2-expressing cells coexpress the panhematopoietic marker CD45 and the HPCs markers CD34 and CD38. More importantly, NG2 expression in $\mathrm{CB}$ was confined to the CD45 + CD34 + cell subset, indicating that, NG2 is solely expressed in hematopoietic cells, especially in committed HPCs; pDC-progenitors (CD45 + CD34+ $\mathrm{CD} 38+\mathrm{CD} 123++$ and HLADR +$)^{16}$ among others, in line with the occurrence of NG2 + pDC-leukemias lacking MLL. ${ }^{11}$ Taken together, we throw more light on the association between NG2 expression and MLL rearrangements and report preliminary data supporting that NG2 expression does not depend on the particular gene(s) paired to MLL. The fact that CD34+ progenitors, pDC precursors and hESCs express NG2 indicates that NG2 in leukemia might uniquely be expressed in those 
patients where the leukemogenesis process is triggered in a NG2-expressing cell subset, regardless the type of leukemia. More comprehensive clinical, functional, molecular, cytogenetic and immunophenotypic studies are warranted to resolve the current puzzling and confusing significance of NG2 expression in human leukemias.

\section{Acknowledgements}

This work was partially funded by the Andalusian Health Department (Grants Ref: 0029/2006 and 0030/2006 to PM), The Jose Carreras International Foundation against the Leukemia (FIJC05/EDThomas 2006 to PM/CB) and The Spanish Ministry of Health to PM (FIS PI070026). CB is supported by the FIS-ISCIII (Ref: CP07/ 00059). We are indebted to Professor Mel Greaves (ICR, London, UK) for his constructive feedback and support.

\section{Author contribution:}

$\mathrm{CB}$ and RM designed and perfomed experiments and analyzed data. LM performed and analyzed experiments. IP and $\mathrm{MCH}$ provided $\mathrm{CB}$ samples and clinical data. AO contributed key clinical data, analyzed data and revised the manuscript. PM conceived, supported and supervised the work and analyzed the data. CB and PM wrote the paper.

C Bueno ${ }^{1}$, R Montes ${ }^{1}$, L Martín ${ }^{2}$, I Prat $^{3}$, MC Hernandez ${ }^{3}$, $\mathrm{A}^{\prime} \mathrm{Orfao}^{2}$ and P Menendez ${ }^{1}$

${ }^{1}$ Andalusian Stem Cell Bank, Hematopoiesis Department, Instituto de Investigación Biomédica, University of Granada,

${ }^{2}$ Department of Medicine, Cancer Research Centre (IBMCCUSAL/CSIC), University of Salamanca, Salamanca, Spain and ${ }^{3}$ Cord Blood Bank of Málaga, Recinto Hospital Civil, Málaga, Spain

E-mail: pablo.menendez@juntadeandalucia.es

\section{References}

1 Stallcup WB, Cohn M. Correlation of surface antigens and cell type in cloned cell lines from the rat central nervous system. Exp Cell Res 1976; 98: 285-297.

2 Smith FO, Rauch C, Williams DE, March CJ, Arthur D, Hilden J et al. The human homologue of rat NG2, a chondroitin sulphate proteoglycan, is not expressed on the cell surface of normal hematopoietic cells but is expressed by acute myeloid leukemia blasts from poor-prognosis patients with abnormalities of chromosome band 11q23. Blood 1996; 87: 1123-1133.

3 Levine JM, Nishiyama A. The NG2 chondroitin sulphate proteoglycan: a multifunctional proteoglycan associated with immature cells. Persp Dev Neurobiol 1996; 3: 245-259.

4 Behm FG, Smith FO, Raimondi SC, Pui CH, Bernstein ID. Human homologue of the rat chondroitin sulfate proteoglycan, NG2, detected by monoclonal antibody 7.1 identifies childhood acute lymphoblastic leukemias with $\mathrm{t}(4 ; 11)(\mathrm{q} 21 ; \mathrm{q} 23)$ or $\mathrm{t}(11 ; 19)(\mathrm{q} 23 ; \mathrm{p} 13)$ and MLL gene rearrangements. Blood 1996; 87: 1134-1139.

5 Hilden JM, Smith FO, Frestedt JL, McGlennen R, Howells WB, Sorensen $\mathrm{PH}$ et al. MLL gene rearrangement, cytogenetic $11 \mathrm{q} 23$ abnormalities, and expression of the NG2 molecule in infant acute myeloid leukemia. Blood 1997; 89: 3801-3805.

6 Wuchter C, Harbott J, Schoch C, Schnittger S, Borkhardt A, Karawajew $\mathrm{L}$ et al. Detection of acute leukemia cells with mixed lineage leukemia (MLL) gene rearrangements by flow cytometry using monoclonal antibody 7.1. Leukemia 2000; 14: 1232-1238.

7 Borkhardt A, Wuchter C, Viehmann S, Pils S, Teigler-Schlegel A, Stanulla $M$ et al. Infant acute lymphoblastic leukemia-combined cytogenetic, immunophenotypical and molecular analysis of 77 cases. Leukemia 2002; 16: 1685-1690.

8 Schwartz S, Rieder H, Schlaoger B, Burmeister T, Fischer L, Thiel E. Expression of the human homologue of rat NG2 in adult acute lymphoblastic leukemia: close association with MLL rearrangement and a CD10+/CD24+/CD65s+/CD15+ B-cell phenotype. Leukemia 2003; 17: 1589-1595.

9 Zangrando A, Intini F, te Kronnie G, Basso G. Validation of NG2 antigen in identifying BP-ALL patients with MLL rearrangements using qualitative and quantitative flow cytometry: a prospective study. Leukemia 2008; 22: 858-861.

10 Moneypenny CG, Shao J, Song Y, Gallagher EP. MLL rearrangements are induced by low doses of etoposide in human feta hematopoietic stem cells. Carcinogenesis 2006; 27: 874-881.

11 Bueno C, Almeida A, Lucio P, Marco J, García R, de Pablos JM et al. Incidence and characteristics of CD4(+)/HLA DRhi dendritic cell malignancies. Haematologica 2004; 89: 58-69.

12 Meyer C, Schneider B, Reichel M, Angermueller S, Strehl S, Schnittger $S$ et al. Diagnostic tool for the identification of MLL rearrangements including unknown partner genes. Proc Natl Acad SCi USA 2005; 102: 449-454.

13 Eguchi M, Eguchi-Ishimae M, Greaves M. Molecular pathogenesis of MLL-associated leukemias. Int J Hematol 2005; 82: 9-20.

14 Daser A, Rabbitts TH. The versatile mixed lineage leukaemia gene MLL and its many associations in leukemogenesis. Semin Cancer Biol 2005; 15: 175-188.

15 Cobo F, Navarro J, Herrera I, Vivo A, Porcel D, Hernandez C et al. Electron microscopy reveals the presence of viruses in MEFs but neither in human fibroblasts nor in human mesenchymal cells used for hESC maintenance: toward an implementation of microbiological quality assurance program in stem cell banks. Cloning Stem Cells 2008; 10: 1-10.

16 Matarraz S, Lopez A, Barrena S, Fernandez C, Jensen E, Flores J et al. The immunophenotype of different immature, myeloid and B-cell lineage committed CD34+ hematopoietic cells allows discrimination between normal/reactive and myelodysplasic syndrome precursors. Leukemia 2008; 22: 1175-1183.

17 Bueno C, Montes R, García-Castro J, Menendez P. Human embryonic stem cells: a potential system for modelling infant leukaemia harboring MLL-AF4 fusion gene. Drug Discovery Today: Disease Models 2008; 4: 53-60.

18 Levac K, Menendez P, Bhatia M. Intra-bone marrow transplantation facilitates pauci-clonal human hematopoietic repopulation of NOD/SCID/beta2m(-/-) mice. Exp Hematol 2005; 33: 1417-1426.

19 Neudenberger J, Hotfilder M, Rosemann A, Langebrake C, Reinhardt D, Pieters R et al. Lack of expression of the chondroitin sulphate proteoglycanneuron-glial antigen 2 on candidate stem cell populations inpaediatric acute myeloid leukaemia/abn(11q23) and acute lymphoblastic leukaemia/t $(4 ; 11)$. Br J Haematol 2006; 133: 337-344.

20 Hotfilder M, Röttgers S, Rosemann A, Schrauder A, Schrappe M, Pieters $\mathrm{R}$ et al. Leukemic stem cells in childhood high-risk ALL/ $\mathrm{t}(9 ; 22)$ and $\mathrm{t}(4 ; 11)$ are present in primitive lymphoid-restricted CD34+CD19- cells. Cancer Res 2005; 65: 1442-1449.

Supplementary Information accompanies the paper on the Leukemia website (http://www.nature.com/leu) 